PROCEEDINGS OF THE

AMERICAN MATHEMATICAL SOCIETY

Volume 140, Number 3, March 2012, Pages 811-821

S 0002-9939(2011)11058-X

Article electronically published on November 2, 2011

\title{
INTEGRABILITY OF THE SUB-RIEMANNIAN MEAN CURVATURE OF SURFACES IN THE HEISENBERG GROUP
}

\author{
D. DANIELLI, N. GAROFALO, AND D. M. NHIEU
}

(Communicated by Mario Bonk)

\begin{abstract}
The problem of the local summability of the sub-Riemannian mean curvature $\mathcal{H}$ of a hypersurface $M$ in the Heisenberg group, or in more general Carnot groups, near the characteristic set of $M$ arises naturally in several questions in geometric measure theory. We construct an example which shows that the sub-Riemannian mean curvature $\mathcal{H}$ of a $C^{2}$ surface $M$ in the Heisenberg group $\mathbb{H}^{1}$ in general fails to be integrable with respect to the Riemannian volume on $M$.
\end{abstract}

\section{INTRODUCTION}

The local summability of the sub-Riemannian mean curvature $\mathcal{H}$ of a hypersurface $M$ in the Heisenberg group, or in more general Carnot groups, near the characteristic set of $M$, is a problem of interest in several questions in sub-Riemannian geometric measure theory and geometry. For $C^{2}$ surfaces in the first Heisenberg group $\mathbb{H}^{1}$ the fine structure of the characteristic set was studied in the work CHMY]. In this short paper we address the question of whether, given a $C^{2}$ surface $M$ in the Heisenberg group $\mathbb{H}^{1}$, it is always guaranteed that the sub-Riemannian mean curvature $\mathcal{H}$ of $M$ is locally in $L^{1}$ with respect to the standard Riemannian measure on $M$ near the characteristic set $\Sigma_{M}$ of $M$. We recall that $\Sigma_{M}$ is the collection of points of $M$ at which the tangent space coincides with the horizontal plane generated by contact distribution. Our interest in this problem arose in connection with the generalization of a formula of Minkowski to hypersurfaces in the Heisenberg group $\mathbb{H}^{n}$, DGN3. In the proof of such a formula the local summability of $\mathcal{H}$ plays a crucial role. In this respect we mention that some authors (see for instance Lemma 4.3 in [RR], assumption (E) in section 5 in [HP], and Remark 2.47 in [Mo] have in their works assumed such integrability of $\mathcal{H}$, but without providing any evidence in favor of the necessity of such an assumption. It was thus natural for us to wonder whether such local summability of $\mathcal{H}$ near the characteristic set is always true or if there exist examples of surfaces for which such a property fails.

In this paper we construct an example of a $C^{2}$ surface $M \subset \mathbb{H}^{1}$ for which $\mathcal{H}$ fails to be locally in $L^{1}$ with respect to the Riemannian volume on $M$, thus confirming the appropriateness of such a summability assumption. Here is the precise result.

Received by the editors August 25, 2010 and, in revised form, August 31, 2010.

2010 Mathematics Subject Classification. Primary 49Q05; Secondary 53D10.

Key words and phrases. Minimal surfaces, $H$-mean curvature, integration by parts, first and second variation, monotonicity of the $H$-perimeter.

The first author was supported in part by NSF grant CAREER DMS-0239771.

The second author was supported in part by NSF Grant DMS-1001317.

The third author was supported in part by NSC Grant 99-2115-M-008-013-MY3. 
Proposition 1.1. There exists a $C^{2}$ graph $M=\left\{(x, y, t) \in \mathbb{H}^{1} \mid(x, y) \in \mathbb{R}^{2}, t=\right.$ $u(x, y)\}$ such that its sub-Riemannian mean curvature function $\mathcal{H}$ has the property that

$$
\mathcal{H} \notin L_{l o c}^{1}(M, d \sigma) .
$$

Here, we have denoted by d $\sigma$ the Riemannian volume on $M$.

It is worth mentioning that, as we point out in the proof of Proposition 1.1 our construction does not provide a negative example in the $C^{3}$ category. Since the construction of an example of non-integrability for surfaces whose characteristic set consists of a line is already delicate, it is natural to ask what happens when the characteristic set consists of isolated points. In section 3 we analyze four possible situations of this type and we show that for all of them $\mathcal{H} \in L_{l o c}^{1}(M, d \sigma)$. Although it is tempting to conjecture that this should always be true, the local integrability of $\mathcal{H}$ near an isolated characteristic point remains at this moment an open question.

Finally, in Proposition 3.5 below we prove that if the Riemannian volume is replaced by the sub-Riemannian perimeter measure $d \sigma_{H}$, then for any hypersurface in an arbitrary Carnot group the function $\mathcal{H}$ is locally integrable.

\section{Proof of Proposition 1.1}

We recall that the Heisenberg group $\mathbb{H}^{1}$ can be identified with $\mathbb{R}^{3}$, with coordinates $(x, y, t)$ and (non-Abelian) group law

$$
(x, y, t) \circ\left(x^{\prime}, y^{\prime}, t^{\prime}\right)=\left(x+x^{\prime}, y+y^{\prime}, t+t^{\prime}+\frac{1}{2}\left(x y^{\prime}-x^{\prime} y\right)\right) .
$$

A basis for the Lie algebra of left-invariant vector fields on $\mathbb{H}^{1}$ associated with such a group law is given by

$$
X=\partial_{x}-\frac{y}{2} \partial_{t}, \quad Y=\partial_{y}+\frac{x}{2} \partial_{t}, \quad T=\partial_{t} .
$$

We notice that the only non-trivial commutation relation satisfied by the vector fields $X, Y$ and $T$ is given by

$$
[X, Y]=T \text {. }
$$

The horizontal bundle $H \mathbb{H}^{1}$ is the union of all planes spanned by the horizontal distribution $\{X, Y\}$. Given a $C^{2}$ surface $M \subset \mathbb{H}^{1}$, the characteristic set $\Sigma_{M}$ is defined as the collection of all points of $M$ at which the tangent plane coincides with $\operatorname{span}\{X, Y\}$.

We will construct our surface $M$ in the form of an entire graph $t=u(x, y)$ on the $(x, y)$-plane, where $u \in C^{2}\left(\mathbb{R}^{2}\right)$ and $u(0,0)=0$. Since for such a surface a global defining function is given by $\Phi(x, y, t)=u(x, y)-t$, it is well known that $\Sigma_{M}$ is given by those points $(x, y, t) \in M$ for which

$$
X \Phi=u_{x}+\frac{y}{2}=0, \quad Y \Phi=u_{y}-\frac{x}{2}=0 .
$$

In other words, $\Sigma_{M}$ is given by the solutions of the system

$$
\left\{\begin{array}{l}
u_{x}(x, y)-\frac{y}{2}=0 \\
u_{y}(x, y)+\frac{x}{2}=0 \\
t=u(x, y)
\end{array}\right.
$$


Upon identifying $\mathbb{R}^{2} \times\{0\}$ with $\mathbb{R}^{2}$, we see that the projection $\Sigma_{M}^{\prime}$ of $\Sigma_{M}$ onto the $(x, y)$-plane is given by

$$
\Sigma_{M}^{\prime}=\left\{(x, y) \in \mathbb{R}^{2} \mid u_{x}+\frac{y}{2}=0, u_{y}-\frac{x}{2}=0\right\} .
$$

The sub-Riemannian mean curvature of $M$ is independent of $t$ and is given by the formula

$$
\mathcal{H}(x, y)=\frac{\left(u_{x}+\frac{y}{2}\right)^{2} u_{y y}-2\left(u_{x}+\frac{y}{2}\right)\left(u_{y}-\frac{x}{2}\right) u_{x y}+\left(u_{y}-\frac{x}{2}\right)^{2} u_{x x}}{\left(\left(u_{x}+\frac{y}{2}\right)^{2}+\left(u_{y}-\frac{x}{2}\right)^{2}\right)^{\frac{3}{2}}} .
$$

Of course, the quantity on the right-hand side of (2.3) is a priori well-defined only outside the set $\Sigma_{M}^{\prime}$.

In order to establish Proposition 1.1 we are going to choose the function $u$ in the form

$$
u(x, y)=\frac{x y}{2}+g(y)
$$

with $g \in C^{2}(\mathbb{R})$ and satisfying the additional assumptions

$$
g(0)=g^{\prime}(0)=0 .
$$

(We note that if the function $u$ took the form $u(x, y)=\frac{x y}{2}+g(x)$, then we would have $\mathcal{H} \equiv 0$, and the surface $M$ would be minimal.) With the choice (2.4) in place, we have

$$
u_{x}+\frac{y}{2}=y, \quad u_{y}-\frac{x}{2}=g^{\prime}(y),
$$

and since $g^{\prime}(0)=0$ we easily see that

$$
\Sigma_{M}^{\prime}=\left\{(x, 0) \in \mathbb{R}^{2} \mid x \in \mathbb{R}\right\} .
$$

Furthermore, the condition $g(0)=0$ guarantees that the characteristic set is given by the line

$$
\Sigma_{M}=\left\{(x, 0,0) \in \mathbb{R}^{3} \mid x \in \mathbb{R}\right\} .
$$

To compute $\mathcal{H}$ we first observe that $u_{x x}=0, u_{x y}=1 / 2$ and $u_{y y}=g^{\prime \prime}(y)$. Therefore, outside the set $\Sigma_{M}^{\prime}$, we obtain from (2.3),

$$
\mathcal{H}(x, y)=\frac{y^{2} g^{\prime \prime}(y)-y g^{\prime}(y)}{\left(y^{2}+g^{\prime}(y)^{2}\right)^{\frac{3}{2}}}=\frac{\frac{g^{\prime \prime}(y)}{y}-\frac{g^{\prime}(y)}{y^{2}}}{\left(1+\frac{g^{\prime}(y)^{2}}{y^{2}}\right)^{\frac{3}{2}}}=\frac{A^{\prime}(y)}{\left(1+\frac{g^{\prime}(y)^{2}}{y^{2}}\right)^{\frac{3}{2}}},
$$

where we have let

$$
A(y) \stackrel{\text { def }}{=} \frac{g^{\prime}(y)}{y} .
$$

We notice that, since by the assumption $g^{\prime}(0)=0$ we have

$$
\lim _{y \rightarrow 0} A(y)=\lim _{y \rightarrow 0} \frac{g^{\prime}(y)}{y}=g^{\prime \prime}(0),
$$

then for any given point on $\Sigma_{M}^{\prime}$ there exists a neighborhood of that point in which the denominator of the last term in (2.5) does not vanish. As a consequence, the local summability of $\mathcal{H}$ on $M$ with respect to the Riemannian volume

$$
d \sigma=\sqrt{1+u_{x}^{2}+u_{y}^{2}} d x \wedge d y
$$

is equivalent to the local summability of $A^{\prime}(y)$ with respect to $d x d y$. 
At this point we pause to observe that, under the additional hypothesis that $g \in C^{3}(\mathbb{R})$, our construction does not produce an example of non-summability. For this, it suffices to observe that, if $g \in C^{3}(\mathbb{R})$, then Taylor's formula gives

$$
\lim _{y \rightarrow 0} A^{\prime}(y)=\lim _{y \rightarrow 0}\left\{\frac{g^{\prime \prime}(y)}{y}-\frac{g^{\prime}(y)}{y^{2}}\right\}=\frac{g^{\prime \prime \prime}(0)}{2} .
$$

We thus conclude that, when $g \in C^{3}(\mathbb{R})$, then $A^{\prime} \in L_{\text {loc }}^{1}\left(\mathbb{R}^{2}, d x d y\right)$.

Back to our example, we now make a specific choice of $g$. To define $g$ we use the formula (2.6). Precisely, we fix $b>0$ and for $y>0$ we set

$$
A(y)=\int_{y}^{b} \frac{\sin (\ln \eta)}{\ln \eta} \frac{d \eta}{\eta}=\int_{\ln \frac{1}{b}}^{\ln \frac{1}{y}} \frac{\sin z}{z} d z,
$$

where we have made the substitution $z=-\ln \eta$. It is clear that

$$
L=\lim _{y \rightarrow 0^{+}} A(y)=\int_{\ln \frac{1}{b}}^{\infty} \frac{\sin z}{z} d z<\infty .
$$

Again for $y>0$ we now let

$$
G(y)=\int_{0}^{y} \eta A(\eta) d \eta
$$

and finally define $g: \mathbb{R} \rightarrow \mathbb{R}$ by setting $g(y)=G(|y|)$. We note that $G \in C^{2}((0, \infty))$ and that furthermore, if we set

$$
G(0)=G^{\prime}(0)=0,
$$

then $G \in C^{2}([0, \infty))$, and $G^{\prime \prime}(0)=L$. As a consequence, $g \in C^{2}(\mathbb{R}), g(0)=g^{\prime}(0)=$ 0 , and $g^{\prime \prime}(0)=L$.

Having made our choice of $g$, to reach the desired conclusion we now go back to the last term in (2.5), and observe that with our choice we have $A^{\prime} \notin L_{l o c}^{1}\left(\mathbb{R}^{2}, d x d y\right)$. We have in fact for any $0<\epsilon<1$,

$$
\int_{0}^{\epsilon}\left|A^{\prime}(y)\right| d y=\int_{0}^{\epsilon} \frac{|\sin (\ln y)|}{|\ln y|} \frac{d y}{y}=\int_{\ln \frac{1}{\epsilon}}^{\infty} \frac{|\sin z|}{z} d z=\infty .
$$

\section{ISOLATED CHARACTERISTIC POINTS}

As the proof of Proposition 1.1 shows, the construction of an example of nonintegrability for surfaces in $\mathbb{H}^{1}$ whose characteristic set consists of a line is somewhat delicate. It is thus natural to wonder what happens when the characteristic set $\Sigma_{M}$ consists of isolated points. In this respect we must say that our attempts at constructing an example of non-integrability of $\mathcal{H}$ in this situation have not been met with success, and therefore it would be tempting to make the following conjecture:

Conjecture. Let $M \subset \mathbb{H}^{1}$ be a $C^{2}$ immersed surface. If its characteristic set $\Sigma_{M}$ consists of isolated points, then $\mathcal{H} \in L_{\text {loc }}^{1}(M, d \sigma)$.

Although this conjecture remains an open question, in this section we provide some interesting evidence in its favor. In what follows we exclusively consider the case of an isolated characteristic point on the surface $M$. Using left-translations, we can without loss of generality assume that such a point be the origin $(0,0,0)$, and that $M$ be locally given by the equation

$$
t=u(x, y), \quad(x, y) \in U,
$$


with $U \subset \mathbb{R}^{2}$ being a sufficiently small neighborhood of $(0,0), u \in C^{2}(U)$, and $u(0,0)=0$. The following simple result provides the first evidence in favor of the conjecture.

Proposition 3.1. Suppose that $M$ has cylindrical symmetry near the isolated characteristic point $(0,0,0)$, i.e., that

$$
u(x, y)=f\left(\frac{x^{2}+y^{2}}{4}\right)
$$

Then $\mathcal{H} \in L^{1}(M, d \sigma)$.

Proof. We use formula (3.14) in [DGN2], which gives for the horizontal mean curvature

$$
\mathcal{H}=-\frac{2 s f^{\prime \prime}(s)+f^{\prime}(s)\left(1+f^{\prime}(s)^{2}\right)}{2 \sqrt{s}\left(1+f^{\prime}(s)^{2}\right)^{3 / 2}}, \quad s=\frac{x^{2}+y^{2}}{4} .
$$

From such a formula it is immediate to verify that, given $B(r)=\left\{(x, y, t) \in \mathbb{H}^{1} \mid\right.$ $\left.\left(x^{2}+y^{2}\right)^{2}+16 t^{2}<r^{4}\right\}$, then

$$
\int_{M \cap B(r)}|\mathcal{H}| d \sigma \cong \int_{x^{2}+y^{2}<r^{2}} \frac{d x d y}{\sqrt{x^{2}+y^{2}}}<\infty .
$$

Returning to the general situation (3.1) we remark that if we consider the two functions

$$
p=u_{x}+\frac{y}{2}, \quad q=u_{y}-\frac{x}{2}, \quad(x, y) \in U
$$

then $p_{y}=u_{x y}+\frac{1}{2}, q_{x}=u_{x y}-\frac{1}{2}$, and so

$$
p_{y}(x, y) \neq q_{x}(x, y), \quad \text { at every }(x, y) \in U \text {. }
$$

This means that, in particular, $p_{y}$ and $q_{x}$ cannot vanish simultaneously at any point $(x, y) \in U$. Since we are assuming that $(0,0,0)$ is an isolated characteristic point of $M$, by possibly shrinking the open set $U$ we can assume that $\Sigma_{M}^{\prime} \cap U=\{(0,0)\}$.

Suppose that $p_{y}(0,0) \neq 0$ (if this is not the case, then it must be $q_{x}(0,0) \neq 0$, and we argue similarly). By the implicit function theorem the zero set of $p$ is locally described as $y=f(x)$ in a neighborhood of $x=0$.

We now consider the situation in which $u$ is of the form

$$
u(x, y)=\frac{x y}{2}+F(x)+G(y),
$$

where $F, G \in C^{2}(I)$ for $I=(-\delta, \delta)$ and some small $\delta>0$, and

$$
F(0)=G(0)=F^{\prime}(0)=G^{\prime}(0)=0 .
$$

We note that for surfaces in the form (3.2) it is relatively easy to describe the local structure of the characteristic set around the isolated characteristic point $(0,0,0)$. We note in fact that in the present case we have

$$
p=y+F^{\prime}(x), \quad q=G^{\prime}(y),
$$

and thus the zero set of $p$ is the curve passing through the origin:

$$
\mathcal{C}=\left\{(x, y) \mid x \in I, y=-F^{\prime}(x)\right\} .
$$

On the other hand, from our assumption that $G^{\prime}(0)=0$, we see that all points on the straight line $\{(x, 0) \mid x \in \mathbb{R}\}$ are on the zero set of the function $q$. However, if $y_{0} \in \mathbb{R}$ is such that $G^{\prime}\left(y_{0}\right)=0$, then also the line $\left\{\left(x, y_{0}\right) \mid x \in \mathbb{R}\right\}$ is contained in the zero set of $q$. The assumption that $(0,0,0)$ be isolated translates into the 
fact that in a sufficiently small neighborhood of $x=0$ the curve $\mathcal{C}$ has no other intersections with the zero set of $q$. If the zeros of the function $G^{\prime}$ do not accumulate at $y=0$, this means that $\mathcal{C}$ has no other intersection with the $x$-axis. Now, three situations can typically occur: 1) $\mathcal{C}$ is transverse to the $x$-axis; 2) $\mathcal{C}$ is tangent to the $x$-axis with a contact of finite order; 3$) \mathcal{C}$ is tangent to infinite order to the $x$-axis. In each case we show that $\mathcal{H} \in L_{\text {loc }}^{1}(M, d \sigma)$, thus providing some further evidence in favor of the conjecture.

For convenience we now set

$$
f(x)=F^{\prime}(x), \quad g(y)=G^{\prime}(y) .
$$

Then $f, g \in C^{1}(I)$, and it is easy to see from (2.3) that for a function $u$ of the form (3.2) the horizontal mean curvature is given by

$$
\mathcal{H}(x, y)=\frac{g^{\prime}(y)(y+f(x))^{2}-g(y)(y+f(x))+g(y)^{2} f^{\prime}(x)}{\left((y+f(x))^{2}+g(y)^{2}\right)^{3 / 2}} .
$$

We begin by analyzing a situation in which the curve $\mathcal{C}$ is transverse to the $x$-axis.

Proposition 3.2. Suppose that $f^{\prime}(0) \neq 0$ and $g(y)=y$. Then, $\mathcal{H} \in L_{\text {loc }}^{1}(M, d \sigma)$.

Proof. From (3.3) we easily obtain

$$
|\mathcal{H}|=\left|\frac{(y+f(x))^{2}-y(y+f(x))-y^{2} f^{\prime}(x)}{\left((y+f(x))^{2}+y^{2}\right)^{\frac{3}{2}}}\right| \leq \frac{C}{\sqrt{(y+f(x))^{2}+y^{2}}},
$$

where in (3.4) we have used the fact that $\left|f^{\prime}\right| \leq C$ on $I$. To prove that $\mathcal{H} \in$ $L^{1}(M, d \sigma)$ it thus suffices to show that

$$
\int_{B(0, \delta)} \frac{d x d y}{\sqrt{(y+f(x))^{2}+y^{2}}}<\infty .
$$

The assumption $f^{\prime}(0) \neq 0$ implies that, by possibly restricting the interval $I=$ $(-\delta, \delta)$, the function $f$ is invertible on $I$. We thus consider the change of variables $(x, y)=\Phi(v, w)=\left(f^{-1}(v-w), w\right)$, which is invertible with inverse $(v, w)=$ $\Phi^{-1}(x, y)=(y+f(x), y)$. The Jacobian of the non-singular transformation $\Phi$ is $\left|J_{\Phi}(v, w)\right|=\left|\left(f^{-1}\right)^{\prime}(v-w)\right|$. Since $f^{\prime}$ is $C^{1},\left|J_{\Phi}\right|$ is bounded in $\Omega=\Phi^{-1}(B(0, \delta))$. Notice that $(0,0) \in \Omega$. Hence,

$$
\int_{B(0, \delta)} \frac{1}{\sqrt{(y+f(x))^{2}+y^{2}}} d x d y=\int_{\Omega} \frac{\left|J_{\Phi}(v, w)\right|}{\sqrt{w^{2}+v^{2}}} d w d v<\infty .
$$

Next, we analyze a situation in which the curve $\mathcal{C}$ has a finite order of contact with the $x$-axis.

Proposition 3.3. Suppose $f(x)=x^{m}, g(y)=y^{k}$, where $m$ and $k$ are positive integers. Then $\mathcal{H} \in L_{\text {loc }}^{1}(M, d \sigma)$.

Proof. The case $m=k=1$ is contained in Proposition 3.2 . Hence, we assume that either $m \geq 2$ and $k \geq 1$ or $m \geq 1$ and $k \geq 2$. In this situation, we compute the quantities $p$ and $q$. We have

$$
\begin{gathered}
p=u_{x}+y / 2=y+x^{m}, \quad q=u_{y}-x / 2=y^{k}, \\
u_{x x}=m x^{m-1}, \quad u_{x y}=\frac{1}{2}, \quad u_{y y}=k y^{k-1} .
\end{gathered}
$$


From these equations and (3.3) we obtain

$$
|\mathcal{H}|=\left|\frac{k y^{k-1}\left(y+x^{m}\right)^{2}-\left(y+x^{m}\right) y^{k}+m x^{m-1} y^{2 k}}{\left(\left(y+x^{m}\right)^{2}+y^{2 k}\right)^{3 / 2}}\right| \leq \frac{C}{\sqrt{\left(y+x^{m}\right)^{2}+y^{2 k}}}
$$

for $(x, y) \in B(0,1)$. Hence, it suffices to show that

$$
I=\int_{B(0,1)} \frac{d x d y}{\sqrt{\left(y+x^{m}\right)^{2}+y^{2 k}}}<\infty .
$$

By elementary considerations we can reduce this to proving the finiteness of the integral on the region $B(1)^{+}=\{(x, y) \in B(0,1) \mid x, y>0\}$. With this objective in mind, we make the change of variables

$$
(x, y)=\Phi(r, \theta)=\left(r^{\frac{1}{m}} \cos ^{\frac{1}{m}} \theta, \quad y=r \sin \theta\right),
$$

whose Jacobian is

$$
\left|J_{\Phi}(r, \theta)\right|=\frac{1}{m} r^{\frac{1}{m}} \cos ^{\frac{1}{m}-1} \theta .
$$

We now observe that: (i) $B(1)^{+} \subset E(1)^{+} \stackrel{\text { def }}{=}\left\{(x, y) \mid x, y>0, x^{2 m}+y^{2}<1\right\}$, and (ii) $\Phi^{-1}$ maps $E(1)^{+}$onto $(0,1) \times(0, \pi / 2)$ injectively. It will therefore suffice to prove the finiteness of the integral on $E(1)^{+}$. But one easily has

$$
\begin{aligned}
\int_{E(1)+} \frac{d x d y}{\sqrt{\left(y+x^{m}\right)^{2}+y^{2 k}}} & =\frac{1}{m} \int_{0}^{\pi / 2} \int_{0}^{1} \frac{r^{\frac{1}{m}-1} \cos ^{\frac{1}{m}-1} \theta}{\sqrt{(\sin \theta+\cos \theta)^{2}+r^{2(k-1)} \sin ^{2 k} \theta}} d r d \theta \\
& \leq \frac{1}{m} \int_{0}^{\pi / 2} \frac{d \theta}{|\sin \theta+\cos \theta| \cos ^{1-\frac{1}{m}} \theta} \int_{0}^{1} \frac{d r}{r^{1-\frac{1}{m}}}<\infty
\end{aligned}
$$

This establishes the proposition.

Finally, we analyze a situation in which the curve $\mathcal{C}$ has a contact of infinite order with the $x$-axis.

Proposition 3.4. If $f(x)=\exp \left(-x^{-2}\right)$ and $g(y)=y$, then $\mathcal{H} \in L_{l o c}^{1}(M, d \sigma)$.

Proof. We have $f^{\prime}(x)=\frac{2}{x^{3}} f(x)$, and thus from (3.3),

$$
\mathcal{H}=\frac{f(x)^{2}+y f(x)+f^{\prime}(x) y^{2}}{\left((y+f(x))^{2}+y^{2}\right)^{3 / 2}}=\frac{f(x)^{2}+y f(x)+\frac{2}{x^{3}} f(x) y^{2}}{\left((y+f(x))^{2}+y^{2}\right)^{3 / 2}} .
$$

Since as a function of $(x, y)$ we have $\mathcal{H} \in C\left(\mathbb{R}^{2} \backslash\{(0,0)\}\right)$, to show that $\mathcal{H}$ is locally integrable it suffices to show that

$$
\int_{B\left(0, \frac{1}{2}\right)}|\mathcal{H}| d x d y<\infty
$$

We now observe that

$$
|\mathcal{H}| \leq \frac{f(x)^{2}+|y| f(x)+\frac{2}{|x|^{3}} f(x) y^{2}}{\left((y+f(x))^{2}+y^{2}\right)^{3 / 2}}
$$

and that furthermore we have for any $0<\varepsilon<1$,

$$
(y+f(x))^{2}+y^{2} \geq 2 y^{2}+f(x)^{2}-\varepsilon f(x)^{2}-\frac{1}{\varepsilon} y^{2}=(1-\varepsilon) f(x)^{2}+\left(2-\frac{1}{\varepsilon}\right) y^{2} .
$$


If we choose $\varepsilon=\frac{3}{4}$, then this inequality gives

$$
(y+f(x))^{2}+y^{2} \geq \frac{1}{4}\left(y^{2}+f(x)^{2}\right) .
$$

To prove (3.7) it will thus suffice to show that

$$
\int_{B\left(0, \frac{1}{2}\right)} \frac{f(x)^{2}+|y| f(x)+\frac{2}{|x|^{3}} f(x) y^{2}}{\left(y^{2}+f(x)^{2}\right)^{3 / 2}} d x d y<\infty .
$$

Since the integrand in (3.8) is even both in $x$ and $y$, it thus suffices to prove that

$$
\int_{B^{+}} \frac{f(x)^{2}+y f(x)+\frac{2}{x^{3}} f(x) y^{2}}{\left(y^{2}+f(x)^{2}\right)^{3 / 2}} d x d y<\infty
$$

where $B^{+}=B\left(0, \frac{1}{2}\right) \cap \mathcal{Q}^{+}$, and $\mathcal{Q}^{+}$denotes the first (open) quadrant of the plane. Let us now consider the set $E^{+}=\left\{(x, y) \in \mathbb{R}^{2} \mid e^{-\frac{2}{x^{2}}}+y^{2}<\frac{1}{4}\right\} \cap \mathcal{Q}^{+}$. Since for every $x>0$ we have $x^{2} \geq e^{-\frac{2}{x^{2}}}$, it is clear that $B^{+} \subset E^{+}$. Therefore, (3.9) will be true provided that

$$
\int_{E^{+}} \frac{f(x)^{2}+y f(x)+\frac{2}{x^{3}} f(x) y^{2}}{\left(y^{2}+f(x)^{2}\right)^{3 / 2}} d x d y<\infty
$$

The change of variable $\Phi:\left(0, \frac{1}{2}\right) \times\left(0, \frac{\pi}{2}\right) \rightarrow \mathbb{R}^{2}$ defined by

$$
(x, y)=\Phi(r, \theta)=\left(\frac{1}{\sqrt{\log \frac{1}{r \cos \theta}}}, r \sin \theta\right)
$$

is a one-to-one mapping onto $E^{+}$. Its inverse is given by

$$
f(x)=\exp \left(-x^{-2}\right)=r \cos \theta, \quad y=r \sin \theta .
$$

The Jacobian of $\Phi$ is given by

$$
\left|J_{\Phi}(r, \theta)\right|=\frac{1}{2}\left(\log \frac{1}{r \cos \theta}\right)^{-3 / 2} \sec \theta .
$$


Using this mapping we obtain

$$
\begin{aligned}
& \int_{E^{+}} \frac{f(x)^{2}+y f(x)+\frac{2}{x^{3}} f(x) y^{2}}{\left(y^{2}+f(x)^{2}\right)^{3 / 2}} d x d y \\
& =\frac{1}{2} \int_{0}^{\pi / 2} \sec \theta \int_{0}^{1 / 2} \frac{r^{2} \cos ^{2} \theta+r^{2} \sin \theta \cos \theta+2 r^{3} \sin ^{2} \theta \cos \theta(-\log (r \cos \theta))^{3 / 2}}{r^{2}(-\log (r \cos \theta))^{3 / 2}} \frac{d r}{r} d \theta \\
& =\frac{1}{2} \int_{0}^{\pi / 2} \int_{0}^{1 / 2} \frac{\cos \theta+\sin \theta+2 r \sin ^{2} \theta(-\log (r \cos \theta))^{3 / 2}}{(-\log (r \cos \theta))^{3 / 2}} \frac{d r}{r} d \theta \\
& =\frac{1}{2} \int_{0}^{\pi / 2} \int_{0}^{\frac{\cos \theta}{2}} \frac{\cos \theta+\sin \theta+2 v \sec \theta \sin ^{2} \theta\left(\log \frac{1}{v}\right)^{3 / 2}}{\left(\log \frac{1}{v}\right)^{3 / 2}} \frac{d v}{v} d \theta \\
& =\frac{1}{2} \int_{0}^{\pi / 2}(\cos \theta+\sin \theta) \int_{0}^{\frac{\cos \theta}{2}} \frac{1}{\left(\log \frac{1}{v}\right)^{3 / 2}} \frac{d v}{v} d \theta+\int_{0}^{\pi / 2} \sec \theta \sin ^{2} \theta \int_{0}^{\frac{\cos \theta}{2}} d v d \theta \\
& =\frac{1}{2} \int_{0}^{\pi / 2} \sin ^{2} \theta d \theta+\frac{1}{2} \int_{0}^{\pi / 2}(\cos \theta+\sin \theta) \int_{\log \frac{2}{\cos \theta}}^{\infty} \frac{d \xi}{\xi^{3 / 2}} d \xi d \theta .
\end{aligned}
$$

From this computation we conclude that (3.10) holds if and only if

$$
\int_{0}^{\pi / 2}(\cos \theta+\sin \theta) \int_{\log \frac{2}{\cos \theta}}^{\infty} \frac{d \xi}{\xi^{3 / 2}} d \xi d \theta<\infty .
$$

But this is true if and only if

$$
\int_{0}^{\pi / 2} \frac{\cos \theta+\sin \theta}{\left(\log \frac{2}{\cos \theta}\right)^{1 / 2}} d \theta<\infty .
$$

Since the integrand is continuous on $\left[0, \frac{\pi}{2}\right]$, the desired conclusion follows.

We close this paper with a general result which says that if we replace the Riemannian volume on $M$ with the sub-Riemannian one, then in fact $\mathcal{H}$ is locally in $L^{1}$.

Proposition 3.5. Let $\mathbb{G}$ be a Carnot group of arbitrary step, let $M \subset \mathbb{G}$ be a $C^{2}$ manifold of codimension one, and indicate with $d \sigma_{H}$ the sub-Riemannian volume on $M$. Then,

$$
\mathcal{H} \in L_{l o c}^{1}\left(M, d \sigma_{H}\right) .
$$

Proof. For the sake of brevity, we will use without giving further details the notation in [DGN1]. By Proposition 9.9 in DGN1] we have

$$
\mathcal{H}=\sum_{i=1}^{m} \nabla_{i}^{H, M} \bar{p}_{i}
$$

where, with $\nu$ denoting the Riemannian Gauss map on $M$, we have let

$$
p_{i}=\left\langle\nu, X_{i}\right\rangle \text { and } \quad \bar{p}_{i}=\frac{p_{i}}{W},
$$

with

$$
W=\sqrt{p_{1}^{2}+\cdots+p_{m}^{2}} .
$$


From formulas (6.10) and (8.3) in the same paper we have

$$
d \sigma_{H}=W d \sigma,
$$

where we have denoted by $d \sigma$ the differential of the Riemannian volume on $M$.

With the above notation, the characteristic set $\Sigma_{M}$ of $M$ is the closed subset given by

$$
\Sigma_{M}=\{p \in M \mid W(p)=0\} .
$$

We now recall the following result proved by Balogh in the Heisenberg group (see Theorem 1.1 in [B] ) and subsequently extended by Magnani to all Carnot groups (see [Ma]): if $M \subset \mathbb{G}$ is a $C^{1}$ codimension one manifold with characteristic set $\Sigma_{M}$, then

$$
\sigma_{H}\left(\Sigma_{M}\right)=0 .
$$

From (3.11) and using the fact that $\bar{p}_{1}^{2}+\cdots+\bar{p}_{m}^{2}=1$, we obtain on $M \backslash \Sigma_{M}$ :

$$
\begin{aligned}
\mathcal{H} & =\sum_{i} X_{i} \bar{p}_{i}=\frac{1}{W} \sum_{i=1}^{m} X_{i} p_{i}-\frac{1}{W^{2}} \sum_{i=1}^{m} p_{i} X_{i} W \\
& =\frac{1}{W} \sum_{i=1}^{m} X_{i} p_{i}-\frac{1}{W^{3}} \sum_{i, j=1}^{m} p_{i} p_{j} X_{i} p_{j} .
\end{aligned}
$$

Now, since $M$ is of class $C^{2}$, the functions $p_{1}, \ldots, p_{m} \in C^{1}$, and therefore $X_{i} p_{j}$ are continuous functions on $M$. On the other hand, thanks to (3.13), for the functions $p_{i} / W=\bar{p}_{i}$ we have $\left|\bar{p}_{i}\right| \leq 1 \sigma_{H}$-a.e. on $M$. We thus conclude that there exists $C>0$, depending on $M$, such that

$$
|\mathcal{H}| \leq \frac{C}{W}, \quad \sigma_{H} \text {-a.e. on } M .
$$

To reach the desired conclusion we only need to observe that if $K \subset M$ is a compact set, then

$$
\int_{K}|\mathcal{H}| d \sigma_{H} \leq \int_{K} \frac{C}{W} d \sigma_{H}=C \sigma(K)<\infty
$$

\section{ACKNOWLEDGMENTS}

This paper was presented by the third-named author at the Sinica-NCTS/TPE Geometry Seminar in December 2010. We thank Jih-Hsin Cheng, Jenn Fang Hwang and Luca Capogna for stimulating conversations.

\section{REFERENCES}

[B] Z. M. Balogh, Size of characteristic sets and functions with prescribed gradients, J. Reine Angew. Math., 564 (2003), 63-83. MR2021034 (2005d:43007)

[CHMY] J. H. Cheng, J. F. Hwang, A. Malchiodi and P. Yang, Minimal surfaces in pseudohermitian geometry and the Bernstein problem in the Heisenberg group, revised version, 2004, Ann. Sc. Norm. Sup. Pisa, 4 (2005), 129-177. MR2165405 (2006f:53008)

[DGN1] D. Danielli, N. Garofalo and D. M. Nhieu, Sub-Riemannian calculus on hypersurfaces in Carnot groups, Advances in Math., 215 (2007), no. 1, 292-378. MR2354992 (2009h:53061)

[DGN2] - A partial solution of the isoperimetric problem for the Heisenberg group, Forum Math., 20 (2008), no. 1, 99-143. MR2386783 (2009j:53030)

[DGN3] , A sub-Riemannian Minkowski formula, in preparation. 
[HP] R. K. Hladky and S. D. Pauls, Variation of perimeter measure in sub-Riemannian geometry, preprint, 2007.

[Ma] V. Magnani, Characteristic points, rectifiability and perimeter measure on stratified groups, J. Eur. Math. Soc. (JEMS), 8 (2006), no. 4, 585-609. MR2262196 (2007i:53032)

[Mo] F. Montefalcone, Isoperimetric, Sobolev and Poincaré inequalities on hypersurfaces in sub-Riemannian Carnot groups, preprint, 2009.

[RR] M. Ritoré and C. Rosales, Area-stationary surfaces in the Heisenberg group $\mathbb{H}^{1}$, Adv. Math., 219 (2008), no. 2, 633-671. MR2435652 (2009h:49075)

Department of Mathematics, Purdue University, West Lafayette, Indiana 47907

E-mail address: danielli@math.purdue.edu

Department of Mathematics, Purdue University, West Lafayette, Indiana 47907

E-mail address: garofalo@math.purdue.edu

Department of Mathematics, National Central University, Jhongli City, Taoyuan County 32001, Taiwan, Republic of China

E-mail address: dmnhieu@math.ncu.edu.tw 